\title{
COMORBIDADE EM CRIANÇAS E ADOLESCENTES COM TRANSTORNO DO DÉFICIT DE ATENÇÃO
}

\section{Resultados preliminares}

\author{
Isabella Souza', Maria Antônia Serra², Paulo Mattos³, Vanessa Ayrão Franco²
}

\begin{abstract}
RESUMO - Trinta e quatro crianças e adolescentes com idades entre 6 e 16 anos, com diagnóstico de transtorno do déficit de atenção com hiperatividade (TDAH) segundo a DSM-IV, foram examinadas e avaliadas quanto a comorbidade com outros transtornos psiquiátricos através do questionário padronizado P-CHIPS aplicado aos pais. Observou-se expressiva ocorrência de transtornos comórbidos $(85,7 \%)$, sendo o transtorno opositivodesafiador $(20,6 \%)$ e o transtorno de conduta $(39,2 \%)$ os mais comuns. Pelo menos dois outros diagnósticos que não o de TDAH puderam ser observados em $57 \%$ da amostra. A depressão maior pode ser diagnosticada em quatro casos e quadros ansiosos (transtorno de ansiedade generalizada, ansiedade de separação e fobias) foram observados em $34,3 \%$ da amostra. A presença de comorbidade altera de modo significativo o prognóstico da enfermidade e pode sugerir estratégias terapêuticas específicas dependendo de cada caso. A comorbidade frequente também pode sugerir a necessidade do uso de entrevistas diagnósticas que contemplam outros transtornos psiquiátricos.
\end{abstract}

PALAVRAS-CHAVE: atenção, TDAH, comorbidade.

Comorbidity among children and adolescents with attention-deficit disorder: preliminary results

ABSTRACT - Thirty-four children and adolescents with ages from 6 to 16 years having a diagnosis of attentiondeficit hyperactivity disorder (ADHD)according to DSM-IV criteria were examined. Psychiatric comorbidity was evaluated through a standard questionnaire given to parents. There was a significant comorbidity $(85,7 \%)$ in the sample, oppositional-defiant disorder $(20,6 \%)$ and conduct disorder $(39,2 \%)$ being the most common comorbid diagnosis. At least two diagnosis other than ADHD could be found in $57 \%$ of the sample. Major depression was seen in four cases and anxiety states (generalized anxiety disorder, separation anxiety and phobias) were seen in $34.3 \%$ of the sample. Comorbidity modifies prognosis in a significant way and may suggest specific therapeutic interventions according to each case. Frequente comorbidity may suggest the need for diagnostic interviews that covers other psychiatric disorders.

KEY WORDS: attention, ADHD, comorbidity.

O transtorno de déficit de atenção com hiperatividade (TDAH) tem prevalência estimada em torno de 5 a $8 \%$ em diferentes culturas, incluindo o Brasil ${ }^{1,2}$. Estes valores podem variar em função do modo como é feito o diagnóstico (utilizando-se critérios da Associação Psiquiátrica Americana, o Diagnostic and Statistical Manual 4, em entrevista clínica ou estruturada, critérios neuropsicológicos, ou outros.) 0 TDAH associa-se com significativo comprometimento funcional em diversas áreas (acadêmica, profissional, social) e, à medida que o indivíduo cresce, ocorrem também taxas crescentes de comorbidade psiquiátrica ${ }^{3}$. A presença de comorbidade com transtornos comportamentais, transtornos do humor (de- pressão e transtorno bipolar), transtornos de ansiedade, abuso de álcool e drogas aumenta o comprometimento observado em uma significativa parcela destes indivíduos. Estudos de comorbidade revelaram taxas elevadas de ocorrência com outros transtornos psiquiátricos, em especial os transtornos do humor, os transtornos ansiosos e os transtornos disruptivos do comportamento ${ }^{4-7}$. O estudo de genética do TDAH do National Institute of Mental Health ${ }^{5}$ demonstrou de forma clara que os sintomas de TDAH não são meramente expressão do quadro clínico de outros transtornos e representam uma comorbidadereal.

Há algumas evidências que apontam para uma diferença no perfil de comorbidades quando se

Grupo de Estudos do Déficit de Atenção (GEDA) do Instituto de Psiquiatria da Universidade Federal do Rio de Janeiro (UFRJ), Rio de Janeiro RJ, Brasil: ${ }^{1}$ Mestranda em Psiquiatria UFRJ; ${ }^{2}$ Médica do GEDA; ${ }^{3}$ Professor Adjunto da UFRJ, Coordenador do GEDA.

Recebido 17 Outubro 2000, recebido na forma final 21 Dezembro 2000. Aceito 27 Dezembro 2000. 
comparam casos com e sem hiperatividade ${ }^{8}$. Mesmo a prevalência destes tipos, especialmente entre 6 e 11 anos, tende a ser também diferente ${ }^{9}$, sendo o grupo sem hiperatividade menos encontrado; esta tendência entretanto se atenua à medida que se avaliam grupos etários mais velhos. O próprio diagnóstico do subtipo sem hiperatividade pode ter pouca estabilidade ao longo do tempo ${ }^{10,11}$, embora isto não tenha sido determinado com amostras significativas. Alguns autores, como Barkley ${ }^{8}$ questionam se o tipo "predominantemente desatento" (sem hiperatividade, na nossa amostra) realmente representa um subtipo de TDAH.

Embora a simples presença de hiperatividade e impulsividade possa prever comprometimentos sociais e acadêmicos diferentes (hiperativos são mais socialmente rejeitados e têm mais frequentemente baixa auto-estima, por exemplo) as diferenças de frequência de comorbidade podem representar, ao menos em parte, as diferenças existentes entre os dois subgrupos de TDAH no que diz respeito às regiões neuroanatômicas envolvidas e a patofisiologia. A forma sem hiperatividade (predominantemente desatenta) parece envolver áreas corticais associativas posteriores e/ou alças subcórtico-corticais provavelmente incluindo o hipocampo, enquanto a forma com hiperatividade (predominantemente hiperativa ou mista) envolveria vias pré-frontais-límbicas incluindo o estriado ${ }^{12}$. Os subtipos também se diferenciariam quanto aos perfis neuropsicológicos apresentados, o que poderia contribuir também para uma diferença no desenvolvimento de outros transtornos psiquiátricos ao longo do tempo. Enquanto o subtipo sem hiperatividade se associa com problemas envolvendo a atenção seletiva e a velocidade de processamento de informações, o tipo com hiperatividade se associa a dificuldades na sustentação da atenção ao longo do tempo, com maior vulnerabilidade à distração. Assim, enquanto os portadores de forma sem hiperatividade apresentam piores desempenhos nos testes de destreza viso-motora/velocidade de processamento (tais como o teste substituição de dígitos da bateria Wechsler) e de recuperação mnêmica verbal, aqueles com a forma com hiperatividade não diferem dos controles normais nestes mesmos testes ${ }^{13}$. 0 desempenho acadêmico de portadores da forma sem hiperatividade (não se considerando os problemas comportamentais aos quais a hiperatividade pode estar associada), tende a ser pior.

O objetivo deste estudo foi avaliar a comorbidade de transtornos psiquiátricos em crianças e adoles- centes com diagnóstico de TDAH em tratamento ambulatorial, segundo os critérios da DSM-IV.

\section{MÉTODO}

Este estudo representa a análise preliminar, do tipo observação de casos, de 22 pacientes do sexo masculino e 12 do feminino, com idades entre 6 e 16 anos, em tratamento ambulatorial no GEDA. $O$ grupo foi composto por moradores de diferentes bairros da cidade, frequentadores de escolas públicas, tendo procurado o serviço através de divulgação feita na imprensa (jornal).

Os pacientes eram absorvidos à medida que procuravam o serviço, por ordem de chegada. O critério de inclusão foi atender aos critérios da DSM-IV para TDAH, diagnóstico este obtido através de entrevista semi-estruturada. Foram critérios iniciais de exclusão história de epilepsia, transtornos abrangentes do desenvolvimento e psicoses. Foram posteriormente excluídos do estudo pacientes com retardo mental (apresentando QI inferior a 70 em teste de inteligência).

Todos os pacientes foram submetidos a entrevista clínica inicial, com os pais, por psiquiatra ou interno de medicina experiente; em seguida os pais eram avaliados através de um questionário de perguntas padronizadas (semi-estruturado) de acordo com as diretrizes da DSM-IV, o P-CHIPS desenvolvido por DuPaul e colaboradores ${ }^{14}$. Com o questionário pretendia-se confirmar o diagnóstico clínico de TDAHI e investigar a presença de outros transtornos psiquiátricos. Na experiência de nosso grupo, a administração do P-CHIPS (questionário para pais) em detrimento do CHIPS (versão do mesmo questionário com perguntas para crianças e adolescentes) é mais recomendada pela necessidade da demonstração de comprometimento funcional em diferentes áreas, que é melhor relatado por pais. Entretanto, no caso de abuso de substâncias e transtorno de estresse pós-traumático, utilizamos o CHIPS. No primeiro caso, pela frequência com que o abuso não é conhecido pelos pais; no segundo porque o evento traumático com grande frequência diz respeito aos pais, incluindo maus tratos e abuso sexual. $O$ diagnóstico é sempre feito pela entrevista semi-estruturada, tendo em vista a variabilidade que pode existir entre diferentes entrevistadores quando se utiliza os critérios diagnósticos da DSM-IV sem perguntas padronizadas.

\section{RESULTADOS}

Dentre os 34 pacientes, 2 foram excluídos por apresentarem QI inferior ou igual a 70 e 4 por da- 
Tabela 1. Distribuição entre os sexos e formas do TDAHI.

\begin{tabular}{lccc}
\hline & Homens & Mulheres & Total \\
\hline Hiperativos & 13 & 9 & 22 \\
Não Hiperativos & 4 & 2 & 6 \\
Total & 17 & 11 & 28 \\
\hline
\end{tabular}

dos não confiáveis no questionário (respostas consideradas pouco confiáveis). A amostra analisada consistiu portanto de 28 pacientes, conforme apresentado na Tabela 1. Destes, 6 apresentavam a forma sem hiperatividade (predominantemente desatenta) e 22 a forma com hiperatividade (predominantemente hiperativa/impulsiva ou mista). Na Tabela 2, são apresentados os diagnósticos: um paciente com TDAHI poderia apresentar apenas este diagnóstico ou outros diagnósticos comórbidos.

Do total da amostra, uma minoria apresentava apenas TDAHI; a comorbidade ocorria em 24 casos (85,7\% dos casos). 16 pacientes apresentavam dois outros diagnósticos além do TDAHI. A comorbidade mais comum foi TDAHI associado a transtorno de conduta (11 pacientes).

Quatro pacientes apresentavam quadro depressivo permitindo o diagnóstico de depressão maior segundo a DSM-IV; não foram observados casos de distimia ou transtorno bipolar.

Os quadros ansiosos foram observados em 11 pacientes, sendo 5 casos de transtorno de ansiedade generalizada e 6 casos de transtorno de ansiedade ligada à separação. Os quadros ansiosos eram mais encontrados nos casos com hiperatividade. As fobias foram diagnosticadas em 8 casos, sendo 5 de fobia social e 3 de fobia específica. À semelhança do que ocorria com os demais quadros ansiosos, elas também eram mais encontradas nos casos com hiperatividade.

Os transtornos disruptivos eram fortemente representados na amostra: 7 casos de transtorno opositivo-desafiador (20,6\%) e 11 casos de transtorno de conduta $(39,2 \%)$. Ambos encontravam-se mais presentes na amostra de hiperativos.

Foram ainda observados 1 caso de abuso de substância, 2 casos de enurese, 1 de encoprese, 1 caso de Tourrette e 1 de transtorno de estresse pós-traumático.

Não se observou nenhum caso de transtorno obsessivo-compulsivo.

\section{DISCUSSÃO}

Os resultados preliminares deste estudo com uma amostra considerada pequena frente à prevalência estimada do transtorno na população indicam que além do comprometimento associado aos sintomas básicos de desatenção, hiperatividade e impulsividade, crianças e adolescentes portadores de TDAH com significativa frequência podem apresentar comorbidade com outros transtornos psiquiátricos, o que aumenta potencialmente o seu comprometimento funcional. O pequeno tamanho da amostra não permite tratamento estatístico que forneça dados epidemiologicamente significativos, tratando-se de dados preliminares.

Tabela 2. Diagnósticos segundo a DSM-IV

\begin{tabular}{|c|c|c|c|c|}
\hline Homens $\mathrm{H}+$ & Homens $\mathrm{H}-$ & Mulheres $\mathrm{H}+$ & Mulheres H- & Diagnóstico \\
\hline 1 & 2 & 1 & 0 & Apenas TDAHI \\
\hline 2 & 0 & 1 & 1 & + Depressão \\
\hline 5 & 0 & 5 & 1 & + Ansiedade \\
\hline 6 & 0 & 1 & 0 & $+\mathrm{TOD}$ \\
\hline 5 & 1 & 4 & 1 & $+\mathrm{T}$. Conduta \\
\hline 5 & 1 & 2 & 0 & + Fobias \\
\hline 0 & 0 & 1 & 0 & + Abuso de Subst. \\
\hline 2 & 0 & 0 & 0 & + Enurese \\
\hline 0 & 0 & 1 & 0 & + TEPT \\
\hline 1 & 0 & 0 & 0 & + Tourrette \\
\hline 0 & 0 & 1 & 0 & + Encoprese \\
\hline
\end{tabular}

$\mathrm{H}+$, com hiperatividade; $\mathrm{H}$-, sem hiperatividade, TOD, transtorno opositivo-desafiador; TEPT, transtorno de estresse pós-traumático. 
A presença de comorbidade modifica de modo significativo o prognóstico do TDAHI, bem como o planejamento das estratégias terapêuticas. Barkley ${ }^{8}$ sugere as seguintes taxas de comorbidade: de 10 a $40 \%$ para transtornos ansiosos, 9 a 32\% para depressão, 20 a $67 \%$ para transtorno opositivo-desafiador e 20 a 56\% para transtorno de conduta.

Observou-se tendência à diferenciação entre as formas com e sem hiperatividade no que tange à presença de comorbidades, o que vai ao encontro da literatura especializada Os transtornos disruptivos, que tendem a ser mais encontrados na forma com hiperatividade e que frequentemente favorecem o encaminhamento a tratamento devem ser entendidos como comorbidade e receber tratamento específico. Não raramente, casos "difíceis" ou "pouco responsivos" de TDAH representam casos de comorbidade que não foi adequadamente diagnosticada.

A presença de depressão, por exemplo, pode sugerir o emprego de antidepressores no tratamento como primeira escolha, ao contrário do que usualmente se recomenda ${ }^{15}$. Também cabe lembrar que é possível que muitos dos casos de depressão sejam na verdade a primeira expressão de um transtorno bipolar: como a faixa etária dos examinandos era mais jovem, é possível que não tivessem ocorrido ainda episódios de mania. A comorbidade entre transtornos bipolares e TDAHI é motivo de inúmeros embates atualmente e as publicações a respeito aumentaram significativamente de número nos últimos anos. Autores que favorecem a importância da ocorrência de mania em episódios melhor delimitados no tempo encontram menos comorbidade entre mania e TDAH do que aqueles que sugerem que a mania possa ser crônica na infância e adolescência. Quadros depressivos foram observados em ambos os grupos.

Os transtornos ansiosos e os transtornos disruptivos do comportamento (transtorno opositivo-desafiador e de conduta) foram significativamente mais encontrados nos casos com hiperatividade, o que vai ao encontro dos resultados de outros autores $^{10,13,16-19}$. Entretanto, é digno de nota a maior prevalência de transtorno de conduta em relação ao transtorno opositivo-desafiador. Embora o pequeno tamanho da amostra por si só possa se associar a frequências que não se comprovarão com números maiores, alguns ítens da DSM-IV talvez tenham levado a diagnósticos de transtorno de conduta em crianças e adolescentes que, na verdade, são melhor diagnosticados como portadores de transtorno opositivo-desafiador. A impressão da equipe que entrevistou a amostra em mais de uma ocasião foi justamente esta. Os critérios para o diagnóstico de transtorno de conduta são apresentados no Anexo e os ítens considerados problemáticos são enumerados a seguir :

1) (item 2. a) Ele costuma mentir para não ter que fazer coisas que não está com vontade de fazer.

2) (item 2. b) Ele frequentemente tenta convencer as pessoas a fazer o que ele quer, do jeito que ele quer.

3) (item 7. a) Ele é brigão.

4) (item 8. b) Ele já se envolveu em problemas por brigar em casa, escola ou na rua.

5) (item 4) Ele já matou aula algumas vezes.

Na nossa amostra, frequentemente vimos pacientes com alguns destes sintomas acima descritos, em combinações variáveis que, entretanto, não apresentavam sintomas mais característicos, tais como precocidade sexual e comportamento sexual inadequado, crueldade com animais, roubos, destruição de propriedade alheia ou violações importantes das expectativas sociais. É possível que cabular aula, brigar e mentir tenham pesos diferentes nas culturas americana e brasileira, sendo considerados comportamentos mais graves na primeira.

Com o aumento da amostra e o seguimento dos casos ao longo do tempo será possível avaliar estes aspectos com maior profundidade, um dos objetivos do estudo.

A presença de transtornos disruptivos é particularmente importante na amostra de portadores de TDAH. Crianças com hiperatividade tendem a ter comportamento mais agressivo e opositivo, têm mais frequentemente baixa auto-estima e sintomas depressivos. O significado destas diferenças, observadas em diferentes estudos, embora não seja claro, permite supor maiores dificuldades inter-pessoais neste tipo. Isto poderia indicar, talvez, a necessidade de um acompanhamento mais próximo e uma investigação frequente deste tipo de dificuldades nos portadores da forma hiperativa.

Sintomas de depressão e ansiedade têm sido denominados de sintomas de internalização por diferentes autores. Crianças com tais sintomas parecem ter piores respostas aos psicoestimulantes ${ }^{20}$ e maiores chances de apresentar transtornos do humor e transtornos de ansiedade na adolescência ou vida adulta ${ }^{11}$.

A presença de comorbidade parece ser significativa no TDAH e pode sugerir a necessidade de entre- 
1. Ele (a) já roubou alguma coisa quando não houvesse ninguém prestando atenção (p.ex. pegou dinheiro, brinquedos, ou coisas dos seus pais, na escola, ou em alguma loja: preencher um cheque que não fosse seu ou usar o cartão de crédito de alguém)?

Se sim, Com que frequência? Pontue apenas se tiver ocorrido mais de uma vez

2. a) Ele (a) costuma mentir para não ter que fazer coisas que não está a fim de fazer? se sim, vá para 3

b) Ele (a) frequentemente tenta convencer as pessoas a fazer o que ele (a) quer ou do jeito que ele (a) quer?

3. Ele (a) já invadiu um carro, um prédio (ou uma casa, um apartamento, uma loja, um posto de gasolina) para roubar ou destruir alguma coisa?

4. Ele (a) já matou aula algumas vezes ou mais?

5. Ele (a) costuma chegar em casa mais tarde do que é permitido ? Se sim Ele (a) fez isto mais do que uma vez por mês? Pontue se ambas as respostas forem positivas

6. Ele (a) já fugiu de casa e ficou a noite toda fora de casa sem voltar? Se sim Ele (a) faz isso com que frequência ? Pontue se mais de uma vez ou se uma vez por um tempo muito longo

7. a) Ele (a) é brigão (ona) ? Se sim, vá para 8

b) Ele (a) ameaça outras pessoas com frequência?

8. a) Crianças se afastam dele (a) porque ele (a) começa brigas ? Se sim, vá para 9

b) Ele (a) já se envolveu em problemas por brigar em casa, na escola ou nas ruas?

Se perguntas 8 a e $8 b$ forem negativas, pule para a pergunta 11

9. Ele (a) já usou alguma arma numa briga? Se sim Com que frequência? Pontue somente se mais de uma vez. O conceito de armas envolve tijolos, tacos, garrafas quebradas, facas ou armas de fogo

10. a) Ele já machucou alguém seriamente numa briga (p.ex. causando um olho roxo, um nariz sangrando, ou que eles fossem obrigados a ir ao médico ou ao hospital) Se sim, vá para 11

b) Ele (a) já feriu alguém sem motivo? (p.ex. beliscou alguém até ficar roxo, ou espetou alguém com objeto ponteagudo)?

11. Ele (a) já tirou coisas dos outros à força (p.ex. roubar uma bolsa ou brinquedos ou roubar uma loja ou posto)?

12. Ele (a) já danificou alguma propriedade (p.ex. quebrar janelas, arranhar carros, furar pneus ou rasgar assentos) ? não conte fogo

13. Ele (a) já pôs fogo em alguma coisa que não deveria ? (p.ex. latas de lixo, coisas em casa, roupas ou animais) ? Se sim Com que frequência? Pontue se mais de uma vez ou se causam danos ou ferimentos extensos

Se nenhuma das respostas acima for positiva, vá para a próxima enfermidade.

14. Ele (a) já machucou ou matou um animal só de brincadeira (p.ex. um cachorro ou gato)?

15. a) Ele (a) já brincou com as partes íntimas - explicar - de alguém sem que a pessoa quisesse?

b) Ele (a) já obrigou alguém a brincar com suas partes íntimas - explicar -?

1 - Critérios Clínicos:

Se 3 ou mais forem positivos, critérios para Transtorno de Conduta foram preenchidos.

2 - Duração:

1) Quando (referência a sintomas evidenciados pelas perguntas 1 a 15) começaram? Anote idade em anos

2) Ele (a) fez ( ) nos últimos 6 meses? Se não, registre a idade com que os sintomas pararam. Se sim, registre idade atual

3) Isso tem acontecido por pelo menos um ano? Registre o número de meses

*Para o diagnóstico de transtorno de conduta atual, é necessário que os sintomas tenham ocorrido por pelo menos 1 ano e estejam presentes nos últimos 6 meses. 
vistas diagnósticas que abordem outros sintomas psíquicos e comportamentais do que aqueles unicamente relacionados àquele transtorno.

\section{REFERÊNCIAS}

1. Rohde L, Biederman J, Busnello E, et al. ADHD in a school sample of brazilian adolescents: a study of prevalence, comorbid conditions and impairments. J Am Acad Child Adolesc Psychiatry 1999;38:716-722.

2. Guardiola A, Fuchs F, Rotta N. Prevalence of attention-deficit hyperactivity disorders in students. Arq Neuropsiquiatr 2000;58: 401-407.

3. Goldman L, Genel M, Bezman R, et al. Diagnosis and treatment of attention deficit / hyperactivity disorder in children and adolescents. JAMA 1998;279:1100-1107.

4. Biederman J, Newcorn J, Sprich S: Comorbidity of attention deficit hyperactivity disorder with conduct, depressive, anxiety and other disorders. Am J Psychiatry 1991;148:564-577.

5. Biederman J, Faraone S, Spencer T, Lapey K. Comorbidity of diagnosis in attention-deficit disorder in Weiss G (ed). Attention deficit hyperactivity disorder. Philadelphia: Saunders, 1992;335-360.

6. Jensen $P$, Shervette R, Xenakis S et al: Anxiety and depressive disorders in attention deficit disorder with hyperactivity: new findings. Am J Psychiatry 1993;150:1203-1209.

7. Jensen P, Martin D, Cantwell D: Comorbidity in ADHD: implications for research, practice and DSM-IV.J Am Acad Child Adolesc Psychiatry 1997;36:1065-1079.

8. Barkley R. Attention-deficit hyperactivity disorder: a handbook of diagnosis and treatment, 2Ed. New York: Guilford, 1998;139-163.

9. Szatmari P, Offord D, Boyle M. Ontario child health study: prevalence of attention deficit disorder with hyperactivity. J Child Psychol Psychiatry 1989;30:219-230.
10. Morgan A, Hynd G, Riccio C et al. Validity of DSM-IV ADHD predominantly inatentive and combined types: relationship to previous DSM diagnoses/subtypes differences. J Am Acad Child Adol Psychiatry 1996;35:325-333.

11. Cantwell D, Baker L. Association between attention deficithyperactivity disorder and learning disorders. In Shaywitz S, Shaywitz $\mathrm{B}$ (eds). Attention deficit disorder comes of age: towards the twentyfirst century. Austin: Pro-Ed, 1992:145-164.

12. Mirsky A. Disorders of attention: a neuropsychological perspective. In Lyon RG, Krasnegor NA (eds), Attention, memory and executive functions. Baltimore: Paul H Brookes, 1996:71-96.

13. Barkley R, DuPaul G, McMurray M. A comprehensive evaluation of attention deficit disorder with and without hyperactivity. J Consult Clin Psychol 1990;58:775-789.

14. DuPaul G, Power T, Anastopoulos A, Reid R. ADHD rating scale-IV. New York: Guilford, 1998.

15. Biederman J, Baldessarini R, Wright V, et al. A doble-blind placebo controlled study of desipramine in the treatment of ADD: III. Lack of impact of comorbidity and family history factors on clinical response. J Am Acad Child Adolesc Psychiatry 1993;32:199-204.

16. Cantwell D, Baker L. Stability and natural history of DSM-III childhood diagnoses. J Am Acad Child Adolesc Psychiatry 1989, 28: 691-700.

17. Hern K \& Hynd G. Clinical differentiation of the attention deficit disorder subtypes: do sensorimotor deficits characterize children with ADDWO ? Arch Clin Neuropsychol 1992;7:77-83.

18. Hynd G, Lorys A, Semrud-Clikeman M, et al. Attention-deficit disorder without hyperactivity: a distinct behavioural and neurocognitive syndrome. J Child Neurol 1991;6:S37-S43.

19. Wheeler J, Carlson C. The social functioning of children with ADD with hyperactivity and ADD without hyperactivity: A comparision of their peer relations and social deficits. J Am Behav Dis 1994;2:2-12.

20. DuPaul G, Barkley R, McMurray M. Response of children with ADHD to methylphenidate: interactions with internalizing symptoms. J Acad Child Adolesc Psychiatry 1994;33:894-903. 